\title{
From Three Ones to Four Ones - Towards a Better Coordinated and Effective AIDS Response
}

\section{Wole Ameyan*}

Solthis, Sierra Leone

\begin{abstract}
The concept and rationale that birthed the Three Ones principle highlighted the need for a unifying national strategic framework, one national coordinating body and a cohesive monitoring and evaluation framework. However, with civil society of People Living with HIVIAIDS (PLHIV) their networks and support groups being increasingly seen as the fulcrum of achieving the bottom lines of adherence and retention of any successful HIV program, their involvement and participation ought to be seen not as support casts but as valid and authentic pivots towards the quest to end AIDS. While the Three Ones has been implemented with varied success, this commentary calls for the inclusion of a fully functional and well-structured civil network of PLHIV as a Fourth One that supports, facilitates and galvanizes a robust PLHIV civil support network and that will triangulate with the three other key principles and ensure that the bottom lines are kept in the front burner.
\end{abstract}

Keywords: Three ones; Four ones; AIDS response; HIV; Civil society; PLHIV networks

\section{Introduction}

In one of the most well thought out policies of the mid-90s that took into account contextual ownership and the need for proper coordination of efforts towards ending AIDS, the Joint United Nations Programme on HIV/AIDS (UNAIDS) in cooperation with the World Bank and the Global Fund to Fight AIDS, Tuberculosis and Malaria and other key donor partners initiated, promoted, advocated and saw to the implementation of the 'Three Ones principle' [1], a strategy that endeavoured to harmonise programmatic leadership, coordination, monitoring and evaluation of HIV programs. The Three Ones gained widespread recognition and acceptance and has sought to promote the most important ideals and principles of any successful HIV program; ownership, alignment and sustainability.

However, in formulating this policy, its proponents failed to look at some of the bottom lines of successful HIV programming. A bottom line being that without the fundamentally significant and centrally prioritized involvement of people living with HIV and AIDS, the ultimate goal of ending AIDS will be foregone. It is concerning that despite assurances and seeming commitment to the GIPA principle (the Greater Involvement of People living with HIV and AIDS) [2] from governments and donors, there is still a gaping lack of realization that any programmatic or policy intervention without PLHIVs acting as the fulcrum is bound to be unsuccessful. Another bottom line is that having harmonised coordination of HIV programs and developed a worthy strategic and M\&E framework, adherence to therapy and retention in care are the most important bottom line goals of HIV programs. It is what leads to people with HIV living longer, healthier lives, and ultimately it is what will break the chain of transmission. This paper makes the case that a strong and viable civil society of PLHIVs is indispensable in any successful HIV programming and they need to be given the same prominence as the other Three Ones.

\section{Discussion}

\section{The three ones principle}

Much of what is today known as the Three Ones principle was decided at an International Conference on AIDS and STIs held in Nairobi, Kenya, in 2003 in which officials from national coordinating bodies and relevant ministries of African nations, major funding mechanisms, multilateral and bilateral agencies, NGOs and the private sector gathered for a consultation to review principles for nationallevel coordination of the HIV/AIDS response [3]. The main aim was to ensure maximum coordination within the international community with a view to leveraging resources, avoiding duplication of effort towards having the maximum impact possible on the global AIDS response.

The principles, which were identified through a preparatory process at global and country levels, took into account opportunities and challenges presented by increasing heterogeneity of funding mechanisms and partnerships and the need to further clarify roles and relationships in this complex dynamic as well as the urgency of local actions and the imperatives of an enabling policy environment include [3]:

THE FIRST ONE (PRINCIPLE I) [4] - One agreed HIV/AIDS Action Framework that provides the basis for coordinating the work of all partners: The action framework in many countries is operated in the form of a national strategic plan for HIV with an operational plan with clear priorities for resource allocation and accountability, making it possible to link priorities, resource flows and outcomes/ results. As advocated and validated during the formulation of the Three Ones principle, a key element of this principle is that the action framework should have an agenda that has widespread buy in, within an environment suitable for agencies, organizations and partners to commit themselves to strive and work within the framework in a manner that is aligned and consistent with country and their own mandates while also allowing for regular and iterative joint progress reviews and consultations. The national strategic plan was also intended

*Corresponding author: Wole Ameyan, Medical Coordinator, Solthis, Sierra Leone, Tel: 07425802519; E-mail: wole.ameyan@solthis.org

Received March 24, 2017; Accepted April 03, 2017; Published April 10, 2017

Citation: Ameyan W (2017) From Three Ones to Four Ones - Towards a Better Coordinated and Effective AIDS Response. J AIDS Clin Res 8: 682. doi: 10.4172/2155-6113.1000682

Copyright: (c) 2017 Ameyan W. This is an open-access article distributed under the terms of the Creative Commons Attribution License, which permits unrestricted use, distribution, and reproduction in any medium, provided the original author and source are credited. 
to provide essential linkages with other strategic national policy documents that provide cross cutting benefits including but not limited to poverty reduction and other national development plans while supporting and enhancing the growing clamour for full participation and engagement of civil society organizations and the other non-public sectors in service delivery.

THE SECOND ONE (PRINCIPLE II) [4] - One National AIDS Coordinating Authority, with a broad based multi-sector mandate: With a nationally verified and accepted strategic plan, complete with interlinkages with other related sectors, there is the need to have the equal of a command and control centre to lead, direct and coordinate the multi-sectoral HIV response. This second principle was formulated with a view to ensuring this. It was envisaged that the central coordinating body would have a formal and legal inaugural mandate with clear roles, responsibilities; reporting and accountability obligations. This second principle requires having a central coordinating with the required programmatic and technical capacities to rally partners while displaying the essential traits of integrity and reliability needed for partners and funding mechanisms within the HIV/AIDS Action Framework to accept their leading role and be assured of the organizational independence to serve their own mandate. Above all, the second principle mandates the National AIDS Coordinating Authority to create a broad, formal, broad based, inclusive and enabling environment for expanding the national responses through information sharing, harmonizing and guiding existing emerging procedures and mechanisms to stimulate and inspire an effective AIDS response.

THE THIRD ONE (PRINCIPLE III) - One agreed country level Monitoring and Evaluation System [4]: With multiple systems for Monitoring and Evaluation (M\&E) as well as the duplication and non-alignment of indicators for measuring performance of countrywide responses resulting in a dearth of data or of poor quality data, which often constrains the monitoring of performance and formulation of evidence-based policies, this third principle had the goal of harmonizing efforts to increase capacity for quality assurance, national oversight and adequate use of M\&E for policy adaptation. This third principle ensures linkages with the other two principles in ensuring that HIV/AIDS Action Framework is accompanied by a robust M\&E plan towards controlling the epidemic under the leadership of the National AIDS Coordinating Authority

In general, while the adoption of the Three Ones principles bolstered efforts to ensuring collective harmonization and alignment with national policies and strategies [5], there have been some challenges in building strategic leadership capacity at the level of the national coordinating bodies. Additionally, global leadership is needed in ensuring that M\&E frameworks are better suited to the production, dissemination, analysis and usage of quality data while facilitating the use of clear, relevant, participatory, measurable and timely indicators. Documented challenges on the Three Ones principle include low level participation in the development of the national strategic framework, incorrect costing of the framework, limited leadership and technical capacity for effective coordination and poor synergy between donors and governments [6].

\section{From three ones to four ones - time for a review and update}

This article calls for a review, update, strengthening and ultimate expansion of the Three Ones principles. The growing quest for civil society engagement, understanding of the need for increased involvement of PLHIV in the planning, implementation and monitoring HIV programs and projects, the impact of a vibrant PLHIV civil society in activating the bottom line of adherence and retention in care necessitates the need for a revision of the scope and application of the Three Ones principle.

Civil society of PLHIV, their networks and support groups are increasingly being seen as the fulcrum of any successful HIV program. Their involvement and participation should therefore be seen not just as support casts but as a valid and authentic pivot for the quest towards ending AIDS. Indeed, PLHIV networks and support groups have been associated with reduced mortality and morbidity, increased retention in care and improved quality of life and implementing support groups as an intervention is seen to have a high impact on morbidity and retention in care on mortality and quality of life of PLHIV [7]. Indeed, the World Health Organization (WHO) and President's Emergency Plan for AIDS Relief (PEPFAR) propose the engagement of support groups as an intervention to address retention and adherence among PLHIV receiving antiretroviral therapy (ART) [8-10]. Many studies have already reported the impact that PLHIV support groups have on increased adherence to ART [11-14]. More strategically and given the shortage of trained health human resource in many sub-Saharan African countries, support groups can play an increasingly larger role with regard to the bottom lines of improved adherence to treatment and retaining HIV-infected persons in care.

There is a strong case to be made here that the stakeholder meeting in Nairobi, Kenya, in September 2003 that birthed the Three Ones principle did not have adequate civil society participation and if it did, such representation was not robust enough to promote and present the need for a PLHIV civil society pillar as a separate but complimentary, obligatory and indispensable mainstay in any HIV strategic response with the goal of ensuring program coordination, monitoring and evaluation using an agreed strategic plan and the bottom line of maintaining adherence, retention in care, improving quality of life, reducing transmission/new infections and reducing morbidity and mortality.

These bottom lines cannot be achieved in good time and in line with Sustainable Development Goals (SDG) timeframes without the fourth one, a fourth principle that supports, facilitates and galvanizes a robust PLHIV civil support network in a coordinated manner to triangulate with the three other key principles and ensure that the bottom lines are kept in the front burner. This article proposes a fourth one thus:

THE FOURTH ONE (PRINCIPLE IV) - One national and fully functional national network of people living with HIV that coordinates the civil society response and complements the national HIV coordinating body: This fourth principle will ensure that civil society efforts in the national response are properly harmonized and complement the national HIV response in a way that is documented and properly monitored. Guiding principles to forge a fully functional national network of PLHIVs should include:

- A national coordinating coalition of PLHIVs with a formally recognized and legal authority to coordinate voluntarily registered civil society organizations, support groups and networks of people living with HIV; advocate for the rights of those infected or affected by HIV; strengthen the capacities of persons living with HIV and the support organizations, groups and networks for greater and meaningful participation.

- Such a mandate will also spell out and define the degree of autonomy of such coalition; specify formal reporting lines and areas of accountability in terms of policy implementation, partner inclusion and programme/development outcomes. 


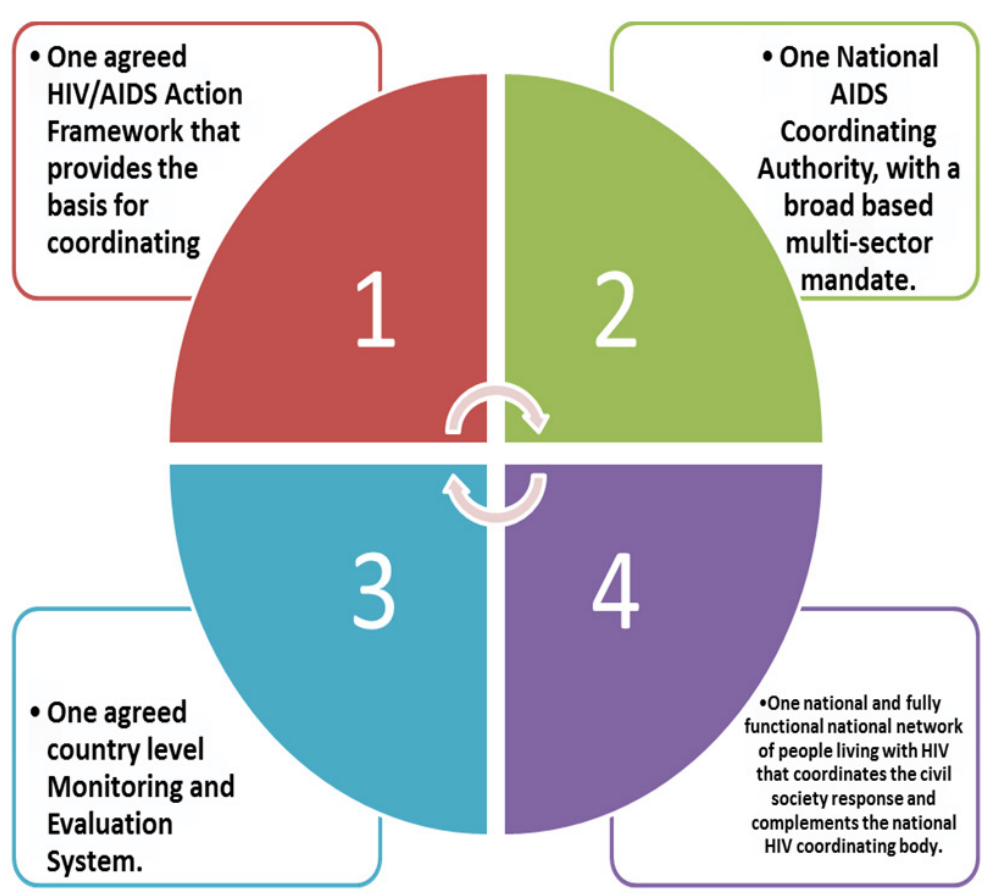

Figure 1: Representation matrix of the Four Ones principle showing the four ones and their interconnectivity.

- The national coordinating coalition shall develop and strive to implement a strategic, operational and M\&E plan that is derived from and takes into account the national strategic action framework. This plan will include clear cut strategies as well as plans and agendas for coordination, linkages and community centred research.

- The national coordinating coalition should be financially independent and be empowered to exercise full and robust technical, functional and structural capacity for developing, implementing, monitoring and evaluating its strategic and operational plan while coordinating resource requests according an inclusive process of agreed priorities.

- Such a national coalition will serve as an umbrella body for all willing PLHIVs irrespective of sex, age, sexual orientation, religion, education or income (Figure 1).

\section{Conclusion}

Overall and despite continued challenges with national ownership and alignment amongst partners and organizations, the Three Ones principle has promoted national level coordination, resource leveraging and strategic priority setting.

The concept of the Three Ones principle has had wide dissemination and while the first principle affirms the growing drive to engage civil society organizations in service delivery, it is time for this engagement to be seen and implemented not as a supporting principle but as a stand-alone, independent and separate norm.

This article therefore calls for a shift from Three Ones to Four Ones to help countries mount sustainable and effective AIDS responses. Rapid progress towards attaining the 90-90-90 targets and the SDGs as it relates to HIV hinges on recognizing the pillar status of PLHIV national networks, the influence and reach on PLHIVs, and ultimately their unassailable significance towards meeting the bottom lines of adherence to treatment, retention in care, improving quality of life, reducing transmission/ new infections and reducing morbidity and mortality.

\section{Disclaimer}

The views expressed in this article are the author's alone and should not be attributed to Solthis or any other institution with which he is associated.

\section{Author Information}

Dr. Wole Ameyan, MIPH is an international HIV specialist with 9 years of progressive experience in technical and strategic HIV programming.

\section{References}

1. WHO (2000) "Three Ones" agreed by donors and developing countries.

2. UNAIDS (1999) From principle to practice: Greater involvement of people living with or affected by HIVIAIDS (GIPA). Best Practice Key Material.

3. Joint United Nations Programme on HIVIAIDS (2004) Three ones key principles. UNAIDS, Geneva 4.

4. UNAIDS (2016) 'Three ones' key principles. Coordination of national responses to HIVIAIDS guiding principles for national authorities and their partners.

5. UNAIDS U (2016) Mainstreaming HIV and AIDS in sectors and programmesAn implementation guide for national responses.

6. Joint United Nations Programme on HIVIAIDS (2005) The "Three Ones" in action: Where we are and where we go from here. World Health Organization.

7. Bateganya M, Amanyeiwe $U$, Roxo $U$, Dong M (2015) The impact of support groups for people living with HIV on clinical outcomes: A systematic review of the literature. J Acquir Immune Defic Syndr 68: S368-S374.

8. World Health Organization (2011) Retention in HIV programmes: Defining the challenges and identifying solutions. Meeting report, Geneva, Switzerland

9. World Health Organization (2008) Operations manual for delivery of HIV prevention, care and treatment at primary health centres in high-prevalence, resource-constrained settings: Edition 1 for field testing and country adaptation.

10. OGAC (2013) Technical considerations provided by PEPFAR technical working groups for FY 2014 COPS and ROPS. OGAC, Washington, DC. 
Citation: Ameyan W (2017) From Three Ones to Four Ones - Towards a Better Coordinated and Effective AIDS Response. J AIDS Clin Res 8: 682. doi: $10.4172 / 2155-6113.1000682$

Page 4 of 4

11. Wouters E, Van Damme W, Van Loon F, Van Rensburg D, Meulemans H (2009) Public-sector ART in the Free State Province, South Africa: Community support as an important determinant of outcome. Soc Sci Med 69: 1177-1185.

12. Achieng L, Musangi $H$, Ong'uti S, Ombegoh E, Bryant L, et al. (2012) An observational cohort comparison of facilitators of retention in care and adherence to anti-retroviral therapy at an HIV treatment center in Kenya. PloS ONE 7: e32727.
13. Elul B, Basinga P, Nuwagaba-Biribonwoha H, Saito S, Horowitz D, et al. (2013) High levels of adherence and viral suppression in a nationally representative sample of HIV-infected adults on antiretroviral therapy for 6, 12 and 18 months in Rwanda. PloS ONE 8: e53586.

14. Mfecane S (2011) Negotiating therapeutic citizenship and notions of masculinity in a South African village. Afr J AIDS Res 10: 129-138. 